CARNETS OE Carnets de géographes

GÉOGRAPHES.

2| 2011

Espaces virtuels

\title{
Entre fiction, simulacre et réalité : les avatars de l'espace virtuel
}

\section{Guy Thuillier}

\section{(2) OpenEdition \\ Journals}

Édition électronique

URL : http://journals.openedition.org/cdg/2674

DOI : $10.4000 / c d g .2674$

ISSN : 2107-7266

Éditeur

UMR 245 - CESSMA

Référence électronique

Guy Thuillier, «Entre fiction, simulacre et réalité : les avatars de l'espace virtuel », Carnets de géographes [En ligne], 2 | 2011, mis en ligne le 02 mars 2011, consulté le 07 mai 2019. URL : http:// journals.openedition.org/cdg/2674; DOI : 10.4000/cdg.2674

La revue Carnets de géographes est mise à disposition selon les termes de la Licence Creative Commons Attribution - Pas d'Utilisation Commerciale - Pas de Modification 4.0 International. 


\title{
Entre fiction, simulacre et réalité : les avatars de l'espace virtuel
}

\author{
GUY THUILLIER \\ Maître de conférences en géographie \\ LISST - Laboratoire Interdisciplinaire Sociétés, Savoirs, Territoires \\ Université de Toulouse II
}

\section{Résumé}

Cet article se propose d'analyser les caractéristiques géographiques des espaces virtuels, à travers une typologie de ces espaces. II existe des liens étroits entre les espaces virtuels réels (jeux vidéo, par exemple), et les espaces virtuels fictionnels décrits dans les oeuvres de science-fiction : la science-fiction influe les créateurs d'univers virtuels réels, et inversement les progrès dans ce domaine inspirent les auteurs de science-fiction. On a donc choisi de s'intéresser autant aux espaces virtuels réels qu'aux espaces virtuels fictionnels imaginés dans les récits de science-fiction. L'espace virtuel est ici pris dans un sens plus large que le seul cyberespace, mais plus précis que l'espace imaginaire. Un espace virtuel implique une sensation d'immersion totale et synesthésique, avec un puissant effet de réel : jeux vidéos, simulateurs, films en 3D, univers transmédiatiques... Ces espaces sont classés en quatre catégories, où la notion de virtuel renvoie à des problématiques différentes : on distinguera ainsi les espaces métaphysiques, cyberpunks, hypergéographiques, et ubiquistes. Certains espaces virtuels peuvent être qualifiés d'hypergéographiques car ils réunissent les quatre propriétés suivantes : la stylisation archétypique, le substrat mythologique, la réminiscence interfictionnelle et la contamination du réel.

\section{Introduction}

Avec le développement des technologies numériques, nous voyons peu à peu prendre forme autour de nous quelques vieux fantasmes de la science-fiction, en particulier l'idée de réalité virtuelle. De nouveaux outils informatiques sont capables de nous propulser dans des univers artificiels de plus en plus riches, qui simulent notre réalité, pour travailler ou pour apprendre. Parfois, ces technologies numériques servent à créer de toutes pièces des mondes imaginaires, sans autre finalité que celle du jeu. Ces espaces virtuels ont été surtout analysés sous des angles technologiques, sociologiques ou psychologiques (Genvo, 2009 ; Fortin \& Tremel, 2009 ; Fortin, Mora \& Tremel, 2006 ; Sussan 2010), philosophiques (Baudrillard, 1991 ; Levy, 1998 ; Virilio, 2010), poétique et narratologiques (Langlet, 2006 ; Genvo, 2006). A ce jour, les espaces virtuels ont donc plus été étudiés pour leur signification sociale et philosophique, ou pour leur fonctionnement diégétique, que pour leurs qualités spatiales intrinsèques. Ces espaces n'ont d'ailleurs que peu 
retenu l'attention des géographes, pour lesquels ils constituent peut-être une dernière frontière, une terra incognita.

Précisons-le d'emblée : si je m'intéresse à ces contrées peu explorées, c'est en tant que géographe, mais aussi en tant qu'auteur de science-fiction, activité qui m'a conduit à imaginer moi-même un univers d'anticipation dans lequel la réalité virtuelle tend à supplanter la vie réelle (Thuillier, 1999). Cette double approche des espaces virtuels, entre géographie et science-fiction, permet en réalité une vision plus riche. En effet, c'est la science-fiction qui a inventé la réalité virtuelle, avant même les premières réalisations concrètes dans ce domaine. Concernant la réalité virtuelle, comme pour d'autres technologies innovantes, la science-fiction a une longueur d'avance sur la science et ses applications technologiques. Si la science-fiction part de la réalité pour élaborer ses univers imaginaires, par des mécanismes d'extrapolation, de projection, d'extension de tendances observables dans le monde réel, il faut noter que bien souvent les ingénieurs s'inspirent en retour des visions de la science-fiction pour développer leurs innovations, dans une dialectique permanente entre science et fiction (Bicaïs, 2007). Comme le souligne Henri Desbois (2006:124) : " avant d'être une métaphore abondamment utilisée par les médias pour désigner plus ou moins tout l'Internet, le cyberespace était d'abord une idée de science-fiction. " C'est pourquoi j'ai choisi, dans ce travail, de m'intéresser non seulement aux embryons d'espaces virtuels existant dans notre réalité (par exemple, les jeux vidéo), mais aussi aux espaces virtuels intrafictionnels, c'est-àdire les espaces virtuels décrits dans les romans et les films de science-fiction. Il s'agit en quelque sorte d'espaces virtuels au carré, des fictions dans la fiction, enchâssés comme des poupées russes dans des univers fictionnels, littéraires ou cinématographiques.

Pierre Levy (1998) nous rappelle justement que virtuel ne s'oppose pas à réel, mais à actuel :

Le mot virtuel vient du latin médiéval virtualis, lui-même issu de virtus, force, puissance. Dans la philosophie scolastique, est virtuel ce qui existe en puissance, et non en acte. Le virtuel est ce qui tend à s'actualiser, sans être passé cependant à la concrétisation effective ou formelle. L'arbre est virtuellement présent dans la graine. En toute rigueur philosophique, le virtuel ne s'oppose pas au réel mais à l'actuel : virtualité et actualité sont seulement deux manières d'être différentes (Levy, 1998 :13).

Il faut cependant admettre que l'espace virtuel (mais réellement existant) d'un jeu comme Second Life, par exemple, possède un degré d'actualisation supérieur à l'espace virtuel du cyberespace (imaginaire) d'un roman de science-fiction, comme la matrice de Matrix ou de Neuromancien. Mais ces espaces virtuels imaginaires requièrent aussi notre intérêt parce qu'ils entretiennent des liens étroits avec les espaces virtuels réels : les espaces virtuels imaginaires des romans de sciencefiction sont en puissance les espaces virtuels réels de demain.

Ces précisions conceptuelles et sémantiques nous amènent, dans un second temps, à préciser ce que nous entendons ici par espace virtuel, en particulier par rapport aux espaces de fiction, aux mondes inventés, aux géographies imaginaires. Dans un sens commun, en effet, la frontière est poreuse entre ces termes. Pour nous, les espaces virtuels ne sont pas simplement des mondes imaginaires. Un espace virtuel implique un puissant effet de réel pour son visiteur : on réservera donc ce terme pour des espaces impliquant une immersion totale, une expérience synesthésique globale, obtenue le plus souvent grâce à de puissants outils de simulation numérique et des interfaces élaborées, comme des lunettes dotées d'écrans internes, des capteurs fixés sur le corps ou les mains pour détecter le mouvement - voire une prise directe sur le cerveau, dans certains 
romans de science-fiction. En général, on se meut dans ces espaces sous la forme d'un avatar numérique, une personnalité virtuelle dont l'aspect et le comportement peuvent être plus ou moins proches de ceux de son propriétaire. Ces caractéristiques distinguent donc ces espaces de simples mondes imaginaires, tels qu'en proposent de nombreuses fictions littéraires ou cinématographiques.

Pour autant, l'espace virtuel ne sera pas réduit au simple cyberespace. Si ce terme a été utilisé dans de multiples acceptions, allant jusqu'à parfois englober tout ce qui se rapporte aux réseaux électroniques, on peut, avec Henri Desbois (2006 : 125), en donner une définition spécifique au champ de la science-fiction : "le cyberespace de la science-fiction est un monde virtuel accessible à travers les réseaux électroniques". Ce cyberespace prend la forme d'une "hallucination consensuelle ", selon William Gibson, l'écrivain de science-fiction inventeur du néologisme cyberspace. On considérera ici que l'accès à des réseaux électroniques via une interface de connexion n'est pas la seule entrée possible dans des espaces que l'on peut qualifier de virtuels. Une expérience de cinéma en 3D avec écran hémisphérique, par exemple, peut être considérée comme une forme d'immersion dans un espace virtuel, sans appel aux réseaux électroniques.

Aujourd'hui, les frontières entre l'imaginaire et le virtuel sont brouillées par le développement des technologies numériques : multiplication des écrans, terminaux numériques portables (smartphones et notebooks), connexion permanente aux réseaux, médias Haute Définition, télévisions 3D à domicile, puissance de calcul en croissance exponentielle, nouvelles interfaces kinesthésiques des consoles de jeu vidéo type Wii ou Kinect, jeux de rôles en ligne massivement multi-joueurs - MMORPG, selon I'acronyme anglais ${ }^{1}$, etc. On voit que toute l'industrie du loisir numérique tend à offrir des expériences de plus en plus totales, permanentes, englobantes, multisupports et multimédia, tendant à décliner des univers sous toutes les formes possibles : livres, films (en 3D), jeux vidéos (interactifs), figurines, jeux de plateaux, réseaux sociaux, etc. L'imaginaire est ainsi lentement colonisé par le virtuel ; la fiction se virtualise à grands pas. Le simple langage, le texte, l'écrit, ne suffisent plus à la génération numérique - les digital natives - qui recherchent des expériences de fictions de plus en plus englobantes, persistantes et multidimensionnelles. Un bon exemple nous en est donné par Avatar, blockbuster en 3D de James Cameron adapté en jeu vidéo et supports multiformes (produits dérivés, jouets, etc.) qui envahissent les gondoles des magasins en période de Noël.

Nous avons donc choisi une définition "moyenne " pour délimiter les espaces virtuels : il s'agit pour nous d'espaces à la fois plus larges que le seul cyberespace (mondes virtuels numériques en réseau), mais plus réduite que l'ensemble des espaces imaginaires (univers fictionnels produit par la littérature et le cinéma traditionnel). On comprend bien, cependant que les frontières ont tendance à se brouiller entre ces catégories, délimitées de manière un peu artificielle au sein d'une sorte de continuum. Où finit l'imaginaire, où commence le virtuel ? Et au sein des espaces virtuels, quand peut-on parler de cyberespace ? Une réponse plus précise à ces questions implique d'examiner de plus près les espaces virtuels, et de se pencher sur les rapports qu'ils entretiennent avec les autres catégories d'espaces dans lesquels ils sont enchâssés, qu'il s'agisse d'espaces fictionnels ou réels.

A quoi ressemblent donc les espaces virtuels ? Comment ont-ils évolué, quelles en sont les grandes figures ? Quels rapports entretiennent-ils avec d'autres dimensions de l'imaginaire : la fiction, la

1 Massive Multi-Players On-line Role Playing Game 
mythologie, le rêve, l'hallucination, le simulacre ? Quel est, enfin, leur rapport avec la réalité ? Notre parcours dans les espaces virtuels, forcément subjectif et sans prétention à l'exhaustivité, se fera à travers une ébauche de typologie. On distinguera ainsi quatre grands types d'espaces virtuels : les métaphysiques, les cyberpunks, les hypergéographiques et les ubiquistes.

\section{Les espaces métaphysiques : fantômes, simulacres et faux-semblants}

Avant de devenir une réalité concrète, les premiers espaces virtuels ont été inventés dans des récits imaginaires. L'espace virtuel est né dans la fiction, avant de déborder vers la réalité. Avant la révolution numérique, les moyens de l'immersion dans ces paléo-espaces virtuels ne sont pas toujours des interfaces informatiques, mais des mécanismes complexes, pas toujours clairement explicités, combinant des hologrammes, des drogues, des outils rituels, voire des marionnettes. Ils sont souvent proches du rêve, de I'hallucination, ou de la transe, qui restent pour l'humanité les premières manifestations balbutiantes d'espaces que l'on peut qualifier de virtuels. Ces récits puisent souvent dans un vieux fond fantastique : l'au-delà, le pays des ombres et des fantômes... L'espace virtuel se loge dans les interstices entre la science et le surnaturel. On retrouve cependant dans ces premiers univers virtuels intrafictionnels les caractéristiques de tout espace virtuel, à savoir l'expérience d'immersion totale, de synesthésie englobante.

Dans L'invention de Morel (1940), de l'écrivain argentin Adolfo Bioy Casarès, le savant Morel, qui vit reclus sur une île, a inventé une machine capable de faire revivre, sous forme d'hologrammes, des personnes disparues. Son île est ainsi peuplée de fantômes recréant l'illusion de la vie, mais toute communication avec eux reste impossible, au grand dam du narrateur, amoureux d'une des chimères qui hante l'île. Ce thème du retour des fantômes, des retrouvailles virtuelles avec de chers disparus peut se retrouver dans des fictions bien plus récentes, assimilables au courant cyberpunk. Dans le film Strange Days, de Kathryn Bigelow (1995), sur un scénario de James Cameron, une technologie nommée SQUID permet, via une sorte de casque sophistiqué, d'enregister sur des mini-discs les flux du cortex cérébral et de les reproduire chez une autre personne. Il est ainsi possible de revivre virtuellement des expériences de son propre passé, ou celles d'autres personnes. Le personnage principal, lui-même dealer d'enregistrement érotiques clandestins, est accroc au SQUID qui lui permet de revivre les moments de bonheur passés avec son ex petite amie - jusqu'au jour où il tombe sur un snuff record : l'enregistrement d'un meurtre en direct....

Parmi les précurseurs de l'imaginaire dans ce domaine des espaces virtuels, on doit bien sûr mentionner l'écrivain états-unien Philip K. Dick, inventeur de multiples espaces virtuels préinformatiques, qui sont plutôt des variations sur le thème du simulacre et du faux-semblant, des jeux autour des rapports entre l'illusion et la réalité. Ses deux plus grands romans contiennent ainsi des espaces virtuels qui apparaissent comme des copies du réel, des reproductions un peu dégradées ou au contraire améliorées de la réalité, et qui sont suscitées par des personnages en situation de privation sensorielle. Dans Le Dieu venu du Centaure, (1965) des colons terriens sur Mars mènent des vies précaires et difficiles de pionniers, reclus sur une base étriquée et sinistre, véritable clapier de l'espace. Ils sont encouragés par les autorités, pour tenir le coup, à profiter de moments d'évasion à travers une expérience de type hallucinatoire, entre le trip procuré par une drogue de synthèse (le D-liss dans la traduction française, ou Can-D en VO), le jeu de rôle halluciné, et l'expérience de possession mystique, en se projetant dans des sortes de poupées, Pat et Walt, sortes d'avatars de Ken et Barbie pour adulte. A travers les poupées Pat et Walt, les colons vivent, par procuration, des vies excitantes et dorées... sur Terre. 
Dans Ubik (1966), un personnage voit la réalité se désagréger peu à peu autour de lui, avant de comprendre qu'il a quitté le monde réel depuis longtemps, et qu'il est prisonnier d'un univers factice, virtuel. En fait, ce personnage est plongé depuis le début du roman, par un attentat, dans un coma halluciné entre la vie et la mort. II évolue dans une sorte projection mentale collective, co-construite par plusieurs autres personnages, allongés comme lui dans des caissons médicalisés qui les maintiennent en vie.

Certes, ce thème d'un univers-simulacre, d'un espace virtuel reproduisant une réalité truquée, factice, reconstituée, ou rêvée, n'est pas absolument nouveau, et l'idée vaguement paranoïaque que le monde qui nous entoure puisse être une gigantesque illusion est presque aussi ancienne que la conscience humaine. On peut évoquer la fameuse allégorie de la caverne de Platon (République, livre VII), qui conçoit le monde comme l'image, la projection, comme dans un théâtre d'ombres, d'une réalité supérieure, abstraite, d'un monde des idées qui est le véritable monde actuel, tandis que notre "réalité " n'en est qu'une sorte de projection virtuelle. On ne peut manquer non plus de citer la fameuse hypothèse de Descartes, qui se demande, dans la démarche de doute systématique entreprise dans ses Méditations Métaphysiques, si le monde tel que nous le percevons existe réellement, ou s'il n'est pas une illusion créée pour nous abuser par quelque « mauvais génie »...

Si l'idée est ancienne, ses avatars dans les fictions modernes doivent cependant beaucoup à l'influence de Philip K. Dick, qui a su articuler ce thème du monde comme illusion aux langages, aux schémas, aux possibilités de la science-fiction. Depuis P. K. Dick, de nombreuses fictions cinématographiques ont revisité ce thème de la réalité comme simulacre virtuel, qui s'impose souvent à des personnages en posture de privation sensorielle (prisonniers, agonisants, convalescents, fous...), dont on manipule l'esprit. En général, quelque faille dans le système finit toujours par dérégler l'illusion, et ces moments où la réalité dérape, où le spectateur comprend que la réalité diégétique n'est qu'une illusion métadiégétique ${ }^{2}$, font tout le sel de ce genre de fictions. Parfois l'écroulement total du monde virtuel amène à un moment de dévoilement, parfois le récit reste cantonné dans cette illusion virtuelle, parfois il joue sur la confusion et les aller-retour entre réalité et illusion, entre espaces diégétique et métadiégétique, soulevant inévitablement d'interminables débats entre les spectateurs pour savoir où commence l'un et où finit l'autre. On retrouve une telle structure dans des films comme Brazil de Terry Gilliam (1985), Total Recall de Paul Verhoven $(1990)^{3}$, Ouvre les yeux d'Alejandro Amenabar (1997), ExistenZ, de David Cronenberg (1999), ou Matrix, des frères Wachowski (1999).

Mais si les espaces virtuels des films cités ci-dessus sont des constructions mentales, obtenues par des manipulations, via des technologies sophistiquées, sur la perception, les souvenirs, l'esprit des personnages piégés dans ces simulacres d'univers, certains espaces virtuels fictionnels sont parfois beaucoup plus low tech. On retrouve alors le simple décor de théâtre, peuplé de figurants-acteurs. Dark City, d'Alex Proyas (1998), ou dans un genre plus réaliste, The Truman Show, de Peter Weir (1998), ou encore Good Bye Lénine de Wolfgang Becker (2003) nous montrent des personnages

2 Nous empruntons ces termes de diégétique et métadiégétique à la théorie narratologique de Gérard Genette (1972). Est diégétique l'ensemble des éléments d'un récit de fiction - parmi lesquels on peut donc compter l'espace dans lequel se déroule cette fiction. Est métadiégétique ce qui se rapporte à un récit dans le récit, une fiction dans la fiction. Un univers virtuel intrafictionnel est donc un univers métadiégétique, inclus dans l'univers diégétique du récit de science-fiction.

3 D'après une nouvelle de P.K. Dick, justement. 
prisonniers d'univers fictifs, reconstitués pour les abuser, et pouvoir les surveiller ou les contrôler, comme des souris dans un labyrinthe. En général, ces espaces virtuels métadiégétiques ne sont autres que la reconstitution de la réalité, qui pour une raison ou pour une autre, se dérobe au personnage. Dans Good Bye Lénine !, la RDA vient de disparaître, mais les proches d'une fervente militante du parti, plongée dans le coma pendant la chute du mur, veulent reconstituer autour d'elle l'illusion que le socialisme existe encore. Dans The Truman Show, Truman, le personnage principal, est à son insu le héros d'une sorte d'émission de télé-réalité qui capte et diffuse sa vie depuis son enfance. Il vit dans une ville artificielle, dont tous les habitants sont des acteurs. Dans Dark City, une ville obscure et inquiétante, évoquant les décors des films expressionnistes allemands de l'entre-deux-guerre, s'avère n'être qu'un simulacre de ville, une reconstitution construite par des extra-terrestres qui y mènent des expériences et des observations sur des humains prisonniers et drogués, inconscients de leur propre captivité.

Qu'il s'agisse de cyberespaces informatiques ou de décors de théâtre en carton pâte, ces univers virtuels intrafictionnels ne sont pas seulement l'occasion de jeux intellectuels et narratifs plus ou moins gratuits. Ils résonnent avec les interrogations contemporaines, propres à l'ère de l'information (Castells, 1999) sur la virtualisation du monde, dans une société saturée d'images plus ou moins truquées, régulièrement accusées de déréaliser le monde, voire de le mener à la catastrophe (Virilio, 2010). Dès 1991, Jean Baudrillard ne se demandait-il pas si la guerre du golfe avait vraiment eu lieu ?

\section{Les espaces cyberpunks : les territoires de l'ordinateur}

Le terme de cyberpunk a été forgé au début des années 80 par la contraction de "cybernétique » et "punk », pour désigner un courant constitué par une poignée d'auteurs de science-fiction, au premier rang desquels William Gibson, et Bruce Sterling (voir l'article d'Henri Desbois dans ce même numéro). Les romans - et plus tard, les films - considérés comme relevant de ce genre décrivent en général des dystopies, univers d'anticipation situés dans un futur proche, présentant souvent certains traits semblables : mégalopoles en déshérence, rongées par les inégalités, la violence et la pollution, livrées au pouvoir de multinationales cupides. Dans ces univers saturés de technologies numériques, les personnages sont souvent des marginaux, plus ou moins rebelles, inspirés par la figure du hacker, du pirate informatique. Une des composantes majeures des romans cyberpunk est bien sûr l'existence à l'intérieur de ces univers diégétiques d'un autre univers métadiégétique sous la forme d'un réseau informatique, plus ou moins évolué, comme la Matrice de Neuromancien (William Gibson, 1984) ou le Métavers du Samouraï virtuel (Neal Stephenson, 1992). C'est naturellement dans ce cyberespace que se déroule une partie des péripéties des romans cyberpunks, qui vont et viennent sans cesse entre les deux niveaux de réalité, diégétique et métadiégétique, et jouent de la confusion entre les deux.

Sans rentrer dans le débat sur la dénomination, le périmètre, la pertinence du genre cyberpunk, il est cependant évident que le développement de l'informatique ouvre au début des années 80 un nouveau champ des possibles à l'imaginaire des espaces virtuels. C'est l'époque des premiers jeux vidéo, des premières consoles et ordinateurs personnels, des premiers réseaux informatiques, etc. Ces innovations marquent les esprits, et l'on assiste à une véritable poussée du fantasme autour de ce qui apparaît encore comme un continent inconnu, une page vierge de l'histoire des sciences et des techniques, qui reste à écrire. 

Dans un premier temps, si l'on considère les espaces virtuels métadiégétiques des productions de cette époque, il semble que l'on puisse, pour qualifier leur contenu, reprendre la célèbre formule de Marshall Mac Luhan (1964) : the medium is the message. Mac Luhan pensait que la forme des média avait une influence plus déterminante sur l'organisation sociale et les rapports sociaux que le contenu véhiculé par ces mêmes médias. En adaptant cette idée à notre propos, on peut constater qu'aux débuts du cyberpunk, la nature et l'organisation du cyberespace métadiégétique est conçu par mimétisme du support de ce cyberespace : la machine informatique elle même. Ainsi, les espaces virtuels engendrés par les ordinateurs ressemblent à... des ordinateurs euxmêmes. Des films comme Tron (1982), ou bien la Matrice de Neuromancien, nous plongent dans un monde abstrait de lignes lumineuses et de blocs géométriques, qui évoque le hardware du système informatique, un labyrinthe de circuits imprimés sur une carte mère, ou encore les interfaces des débuts de l'informatique grand public: graphiques primitifs verdâtres sur l'écran noir des premiers ordinateurs domestiques, façon Apple II ou Atari 1400. L'intéressant, dans le roman de Gibson, qui donne ses lettres de noblesse au cyberpunk par une véritable qualité d'écriture, c'est que les propriétés de l'espace virtuel métadiégétique débordent sur le monde diégétique. L'abstraction, la froideur, la dureté du cyberespace contaminent la dystopie. Le monde diégétique finit par être perçu par les personnages à travers le prisme du virtuel métadiégétique. Ainsi la neige qui tombe sur un paysage urbain est comparée à la neige qui balaie un écran en l'absence de transmission...

Curieusement, cette vision d'un espace virtuel de nature informatique, composé de lignes et de blocs abstraits, inspiré de la matérialité de la machine elle-même (hardware), ou de la nature du code informatique qui la régit (software) se retrouve dans le film Matrix, postérieur de 15 ans à Neuromancien. La Matrice, illusion numérique métadiégétique pourtant très perfectionnée, copie presque parfaite du monde diégétique, se trouve représentée sur l'écran des rebelles qui la surveillent de l'extérieur par un défilé de signes verdâtres, évoquant l'écriture japonaise 4 , mais aussi le code informatique qui modèle la Matrice. Dans Matrix, I' " hallucination consensuelle " qu'est le cyberespace, selon la formule de Gibson, a beau prendre la forme d'une copie hyperréaliste de notre monde, ces colonnes de signes cabalistiques verdâtres nous renvoient à la vraie nature intrinsèque de ce méta-univers, réduit ici à l'architecture invisible qui sous-tend l'illusion, à l'envers du décor : une série de lignes de code informatique.

Avec le développement des technologies numériques dans les années 1990, décennie suivant l'apparition du cyberpunk, les espaces virtuels finissent par quitter le territoire des romans de science-fiction pour peupler notre réalité, et même notre quotidien : la fiction devient réalité. Mais les espaces virtuels réels sont quelque peu différents de leurs précurseurs fictionnels. Ils ne sont plus constitués d'obscurs réservoirs de données abstraites, réservés aux initiés ou aux hackers, comme dans les premières fictions cyberpunks, mais s'ouvrent au commun des mortels, sous la forme d'univers ludiques de plus en plus impressionnants et réalistes. C'est l'heure des jeux vidéo en ligne massivement multi-joueurs (MMORPG), comme Second Life ou World of Warcraft, un univers d'héroic fantasy qui revendique pas moins de 12 millions d'utilisateurs à travers le monde. Outre leur interactivité massive, ces univers ont la caractéristique d'être persistants, c'est-à-dire que contrairement à un jeu vidéo classique, qui n'existe que pendant que l'ordinateur ou la console de jeux qui le génère est allumé, ces mondes continuent à croître et à se modifier, même

4 Cette ressemblance manifeste, selon Henri Desbois (2006 : 133), « le tropisme japonais de la science-fiction actuelle». 

en l'absence d'un joueur donné, qui trouvera donc un univers légèrement différent d'une connexion à l'autre.

On peut qualifier ces MMORPG d'espaces virtuels, car si l'immersion n'est pas (encore) aussi englobante et synesthésique que l'avaient imaginé les précurseurs du cyberpunk - on ne se connecte pas à ces jeux via des prises dans la nuque ou des casques bardés d'électrodes, mais via l'écran d'un simple PC - l'effet d'immersion est tout de même suffisamment puissant, en intensité, en durée, en densité, pour provoquer parfois une véritable déconnexion de la réalité, au point que les psychologues pointent l'apparition d'une nouvelle pathologie dans les sociétés développées : la cyber-dépendance. Les faits divers sordides liés à des affaires de cyber-dépendance font les choux gras de la presse, qui rapporte régulièrement des cas de joueurs morts d'épuisement devant leur écran, après avoir joué pendant des jours sans s'arrêter, ou de parents qui laissent mourir voire assassinent leurs enfants pour pouvoir assouvir leur passion du jeu ${ }^{5}$.

Cette invasion du réel par le virtuel a aussi inspiré les auteurs de science-fiction : ce thème des joueurs absorbés par les mondes virtuels se retrouve dans des romans comme Inner City de JeanMarc Ligny (1996), ou Le dixième cercle, de Guy Thuillier (1999).

Là encore, le rapport à la réalité est au coeur des débats suscités par ces espaces virtuels, en particulier les jeux en ligne, qui suscitent de plus en plus l'attention des chercheurs (par exemple pour World of Warcraft : Coulombe, 2010 ; ou Genvo, 2009 ; pour Second Life, Cayeux \& Guibert, 2007). Le virtuel est perçu (et critiqué) comme une fuite du réel, et rapproché de comportements comme l'addiction aux drogues et autres formes de déviances. Dans une approche plus constructive, l'espace virtuel est appréhendé comme un miroir du réel, une façon de questionner et de tester la valeur de notre modernité. Comment se fait-il qu'il semble parfois plus facile pour les individus de se construire une personnalité ou de trouver des gratifications dans les espaces virtuels, plutôt que de ce côté-ci de l'écran ?

\section{Les espaces hypergéographiques : archétypes, mythes et réminiscences}

A quoi ressemblent donc ces espaces virtuels aujourd'hui, qu'ils soient réels ou intrafictionnels ? Peut-on en faire une géographie?

D'abord, on peut constater que la boîte à outils des espaces virtuels n'est plus sensiblement différente de celle des mondes imaginaires en général. On retrouve dans les espaces virtuels les mêmes traits que dans les univers de fiction, et plus précisément les univers de science-fiction, qui est le genre démiurgique par excellence. En effet, si la fiction crée essentiellement des personnages et des intrigues, qu'elle situe dans des décors existants, la science-fiction invente aussi les espaces où elle déroule ses récits. Dans ses différents courants, qu'il s'agisse du spaceopéra, de la hard-science, de la fantasy, de l'anticipation, du cyberpunk, etc., la science-fiction, ou les "littératures de l'imaginaire ", selon une terminologie en vogue aujourd'hui, a en effet la particularité de proposer au lecteur / spectateur non seulement une intrigue, des personnages imaginaires, mais aussi un univers, plus ou moins proche de notre réalité, et c'est cet effet

5 Par exemple : « Accros à Internet, des parents laissent mourir de faim leur bébé » (20 minutes, 5 mars 2010, http://www.20minutes.fr/article/389016/Monde-Accros-a-Internet-des-parents-laissent-mourir-de-faim-leurbebe.php), consulté le 29/12/2010; ou encore : «Elle tue son fils pour jouer sur Internet » (i20minutes, 22 décembre 2010, http://i.20min.ch/fr/faits\%20divers/25415884/Elle-tue-son-fils-pour-jouer-sur-internet ), consulté le $29 / 12 / 2010$ 
d'étrangeté, de surprise, de découverte, au début de chaque nouveau roman, qui fait tout le sel de ce genre polymorphe (Colson \& Ruaud, 2008; Labbé \& Millet 2001; Langlet, 2006).

Cette proximité des espaces virtuels et des espaces de la science-fiction étant posée, on peut ensuite constater que ces espaces sont souvent des variations autour de grandes catégories d'espaces imaginaires archétypaux, qui trouvent leurs racines dans la mythologie, mais dont les avatars contemporains sont retravaillés en permanence par la culture de masse, dans la littérature, le cinéma, et désormais le jeu vidéo. Nous proposons pour qualifier la géographie de ces espaces virtuels la notion d'hypergéographie. Que signifie ce terme ? On veut par ce néologisme souligner quatre dimensions de ces géographies virtuelles, qu'il faudra expliciter : stylisation archétypique, substrat mythologique, réminiscences interfictionnelles, et contamination du réel. Nous nous servons ici du préfixe hyper- dans ses deux acceptions : le superlatif, car il constitue le procédé principal de la stylisation archétypique; mais aussi l'idée de référence, de lien - comme dans le terme hypertexte - dont procèdent les trois autres dimensions d'une hypergéographie : références à un substrat mythologique, réminiscences interfictionnelles, et contamination du réel. Par ailleurs, le terme d'hypergéographie évoque la notion d'hyperréalité de Jean Baudrillard (1981), avec laquelle l'hypergéographie a bien quelque rapport, car sa quatrième dimension - la contamination du réel - fait parfois accéder l'hypergéographie à une forme d'hyperréalité, c'est-à-dire une forme de simulacre qui finit par remplacer la réalité elle-même.

Pour illustrer ce concept de façon un peu plus concrète, examinons une par une ces quatre dimensions des espaces virtuels hypergéographiques en nous appuyant sur deux exemples concrets : les espaces urbains et les forêts dans la science-fiction.

Les espaces urbains de la science-fiction participent d'une stylisation archétypique dans la mesure où ils présentent souvent des variations autour des représentations stéréotypées que nous nous faisons de la ville. Ils expriment, par des processus d'exagération, de concentration, de cristallisation de certains traits spatiaux, des archétypes plus ou moins conscients de nos catégories spatiales, comme l'a montré Alain Musset (2005) pour Coruscant, capitale de l'empire galactique de la saga cinématographique Star Wars (La guerre des Etoiles). Coruscant est une sorte d'hyper New-York, elle-même ville hypermoderne par excellence, par sa verticalité exacerbée. La verticalité, le gigantisme et la monumentalité sont manifestement hypertrophiées à Coruscant, mais il en va de même pour les autres aspects de l'organisme urbain. Les flux, par exemple, sont magnifiés : les autoroutes urbaines ou les grandes avenues des villes nord-américaines deviennent des flux de véhicules volants en trois dimensions, etc. Les archétypes urbains de la science-fiction sont cependant multiples, voire opposés, comme l'ont montré Eglantine Colon et Irène Langlet (2007) en comparant la vision futuriste de Los Angeles à Solotol, une des villes décrites par lain Banks dans I'Usage des armes, deux villes qui apparaissent comme des modèles antinomiques.

Le substrat mythologique est une composante importante des récits de science-fiction, et pas seulement pour la structuration de l'espace, mais pour l'ensemble du récit, incluant la narration et les personnages (Calvet, 2006). Pour revenir à l'exemple des villes, l'imaginaire urbain d'un film comme Blade Runner, censé se dérouler dans une Los Angeles future, évoque par ses foules bigarrées et cosmopolites, son ambiance sensuelle et interlope, et ses grandes pyramides tronquées aux allures de ziggourat, la biblique Babel / Babylone, ville de I'hybris, de la démesure par excellence (voir également l'article d'Alain Musset dans ce numéro des Carnets).

La réminiscence interfictionnelle marque à l'évidence de nombreuses villes de science-fiction, c'est- 
à-dire qu'outre les grands récits mythologiques, ces espaces s'inscrivent dans une série de références à d'autres fictions ayant elles-mêmes contribué à façonner l'archétype spatial : on ne peut pleinement saisir le sens de ces espaces que par rapport à ces fictions antérieures. Coruscant, par exemple, présente quelque parenté avec Trantor, la ville-planète de Fondation, le classique de la Science-Fiction d'Isaac Asimov, mais aussi avec la Métropolis de Fritz Lang.

La contamination du réel traduit le fait que si l'imaginaire urbain de la science-fiction est particulièrement influencé par quelques villes réelles paradigmatiques (Los Angeles, New York, Tokyo) mais aussi par certaines villes mythiques (Babel / Babylone, en particulier), en retour, cet imaginaire urbain "science-fictionnesque " modèle les représentations que se font les habitants et les édiles de leur propre ville, et conditionne les aménagements, les réalisations urbaines et les pratiques de ces acteurs urbains :

Comme les ingénieurs de I'Internet avaient souvent pour horizon le cyberespace de la science-fiction, les architectes et les urbanistes semblent fréquemment avoir comme modèle une ville virtuelle. Le cas de Tokyo est à cet égard assez révélateur. On a vu que cette ville était déjà en elle-même un référent important des villes de science-fiction. Mais la sciencefiction est aussi une référence explicite pour Tokyo même. (Desbois, 2006 : 136)

Un autre bon exemple d'espace hypergéographique nous est donné par la forêt dans la science fiction. Pierre Jourde (1991) a bien analysé le substrat mythologique, et l'ambivalence fondamentale, des représentations des forêts dans l'oeuvre de J. R. R. Tolkien, l'auteur du Seigneur des Anneaux. La forêt de Mirkwood, où Bilbo est capturé par des araignées géantes, est l'espace obscur de la sauvagerie, de l'animalité, des ténèbres, alors que la forêt des Elfes, la Lothlorien, est un monde lumineux, enchanté, et protecteur, qui évoque la pureté et l'innocence des origines.

Quant à la stylisation archétypique, elle joue aussi à plein pour les forêts des univers de la sciencefiction, qui sont forcément d'une ancienneté et d'une étendue défiant l'imagination. Ainsi, dans Star Wars (épisode $\mathrm{VI}$ : Le retour du Jedi), les scènes de la forêt de la planète Endor, théâtre d'une spectaculaire course-poursuite en moto volantes, ont-elles été filmées en Californie du Nord dans une forêt de séquoias, les plus grands arbres que l'on puisse trouver sur Terre. La forêt d'Endor se présente ainsi comme une futaie aux troncs immenses et droits, archétype de la forêt dans l'imaginaire occidental. Là aussi, cette fiction de la forêt contamine la réalité de la forêt : dans les forêts domaniales de la région parisienne, l'ONF traite les sous-bois plutôt en futaie qu'en taillis, pour complaire aux attentes des visiteurs.

Un autre exemple plus récent, qui mérite que l'on s'y attarde tant il synthétise toutes les caractéristiques hypergéographiques de la forêt, est la forêt qui recouvre la planète Pandora, dans le film Avatar, de James Cameron (2009). Cette forêt renvoie elle aussi à des archétypes mythologiques. Elle est d'abord pour les explorateurs terriens égarés la forêt hostile et obscure, inconnue, la forêt des terreurs primitives où rôde la peur du loup, incarné ici par un quadrupède extra-terrestre à la dentition impressionnante : un hyperloup, en quelque sorte. Mais lorsque le personnage principal est initié aux secrets de cette forêt, elle devient pour lui une sorte de jardin d'Eden, avec ses " bons sauvages " : la tribu des Na'vis, des extra-terrestres qui vivent en harmonie avec une nature généreuse et luxuriante, sorte d'humanité avant la chute, blottie autour de son arbre-maison. Cet arbre géant, dont le tronc creux et les branches servent de refuge à la tribu, renvoie lui aussi à de nombreux mythes comme celui de I'Yggdrasill, l'arbre-monde de la 
mythologie scandinave, qui a par ailleurs inspiré le vaisseau d'Hypérion, célèbre space-opéra de Dan Simmons. Mais cette forêt, quand les Terriens avides viennent la bombarder depuis leurs hyper-hélicoptères de combat pour s'emparer des richesses minières présentes sous le territoire des Na'vis, évoque alors soudain la jungle des guerres états-uniennes du XXe siècle. On retrouve dans ces scènes un topo du film de guerre : le contraste brutal entre l'enfer de la guerre, la mort et la destruction d'une part, et un contexte de beauté naturelle paradisiaque d'autre part. Ce thème de l'enfer au paradis imprègne en particulier certains films sur la guerre du Viet-Nam (Apocalypse Now, Francis Ford Coppola, 1979) ou sur la guerre du Pacifique (La ligne rouge, Terrence Malik, 1998).

L'exemple de la forêt de Pandora nous montre donc comment fonctionne un espace hypergéographique : une combinaison de représentations aux racines bien ancrées dans l'inconscient collectif, agrégation de substrats mythologiques divers - des histoires de loup-garou puisées dans le folklore populaire à l'Eden biblique -, et une stylisation par exagération des traits, des dimensions, des propriétés de cet espace par rapport à son modèle réel implicite. Ainsi Pandora apparaît comme une sorte d'hyper-Amazonie: plus grande, plus impressionnante, plus belle, mais aussi plus dangereuse, et plus menacée. Outre ces référents, Pandora évoque encore dans l'imaginaire collectif interfictionnel d'autres forêts hyperréelles : la jungle des Gl's embourbés au Viet Nam - telle que nous la connaissons par les films de guerre -, ou les forêts luxuriantes des îles du Pacifique. Pandora est donc plus qu'une forêt, une véritable hyperforêt, qui contient d'innombrables autres réminiscences de forêts. Pandora est un espace palimpseste, qui ré-écrit la notion même de forêt par dessus ses versions antérieures et contribue à son tour à façonner I'imaginaire de la forêt tropicale pour le public occidental du début du XXle siècle. Et cet imaginaire déborde sur la réalité, car Pandora valide le dernier critère d'un espace hypergéographique : la contamination du réel.

Ainsi, une ONG a récemment tenté d'utiliser la ressemblance du scénario d'Avatar avec le sort d'une tribu de l'Etat d'Orissa, en Inde, pour attirer l'attention des médias - et de puissants parrains potentiels - sur leur cause :

Comme la tribu des Na'vi qui, dans le film, tente désespérément d'empêcher les humains d'exploiter les ressources minières de leur terre sacrée, les Dongria Kondh sont menacés d'expropriation par une compagnie britannique, Vedanta Resources, qui veut exploiter la bauxite de leur montagne.

"Le drame d'Avatar - si l'on fait abstraction des lémuriens multicolores, des chevaux à longue trompe et des guerriers androïdes - se joue aujourd'hui sur les collines de Niyamgiri en Orissa ", explique Stephen Corry, directeur de l'organisation non gouvernementale (ONG) Survival International, qui défend les peuples indigènes.

Lundi 8 février, I'ONG a publié dans Variety, un magazine américain consacré à l'industrie du spectacle, un appel à James Cameron pour venir en aide à la petite tribu de l'est de I'Inde. " Avatar est une fiction... bien réelle. En Inde, la tribu des Dongria Kondh lutte pour défendre sa terre. (...) Nous avons vu votre film. Maintenant, visionnez le nôtre ", lui demande l'ONG. (BOUISSOU J., 2010, "En Inde, la tribu des Dongria Kondh vit le scénario du film Avatar », Le Monde, 11 février.)

Autre exemple de cette contamination : en février 2010, des militants palestiniens, protestant contre la destruction de plusieurs maisons à Jerusalem-Est, en raison du tracé du mur de 

séparation construit par Israël, ont manifesté déguisés en Na'vis, assimilant leur sort à celui des indigènes de Pandora ${ }^{6}$.

Avatar est en outre un cas particulier et emblématique d'hypergéographie, qui vient marquer un pas supplémentaire dans la virtualisation de l'imaginaire. En effet, l'univers imaginaire de Pandora, atteint une dimension virtuelle : premier grand film en 3D de l'histoire du cinéma, il a bénéficié d'un budget marketing considérable, qui lui a permis d'être vu par des millions de spectateurs à travers le monde. Le film est en outre d'emblée associé à un jeu vidéo et à de nombreux autres produits dérivés. Multisupport, massif, tridimensionnel, interactif : de simple espace imaginaire, décor de cinéma, Avatar accède à un certain niveau de virtualité.

Mais ce film va encore plus loin dans la confusion entre l'imaginaire et le virtuel: l'univers de Pandora peut être interprété comme une métaphore de l'espace virtuel. En effet, il s'agit certes d'une planète, et dans le space-opera classique, type Star Wars, des personnages découvrent de nouvelles planètes par le voyage spatial : on retrouve là une dimension du roman d'aventure ou d'exploration classique. Mais dans Avatar, l'expérience du voyage spatial du début du récit est totalement escamotée, l'action se situant d'emblée dans la base de Hell's Gate, camp retranché sur Pandora, coupé du reste de la planète hostile. La seule façon d'accéder à la dangereuse jungle qui entoure le camp, pour les Terriens, reproduit les modalités d'accès dans le cyberespace telles qu'on les représente dans l'imaginaire cyberpunk: immersion dans un caisson fermé, avec un casque bardé d'électrodes, et mise en catalepsie du corps physique, tandis que l'esprit du voyageur immobile se retrouve aux commandes du corps d'un avatar. Mais contrairement au cyberespace, où le terme avatar désigne couramment un double virtuel de l'opérateur, une identité numérique pour se connecter sur les réseaux, forums ou jeux en ligne, dans le film de James Cameron, les avatars sont des êtres de chair, des clones des créatures extra-terrestres qui peuplent la planète, les Na'vis. Et c'est seulement à travers ces corps, adaptés à la vie sur Pandora, et ressemblant à ceux de leurs interlocuteurs Na'vi, que les Terriens peuvent explorer la forêt de Pandora et entrer en contact avec les indigènes.

On voit comment, dans Avatar, le voyage vers l'espace inconnu d'une planète étrangère reproduit les modalités habituelles de l'accès à l'espace virtuel, via une interface de type réalité virtuelle. Estce si étonnant pour un film qui est conçu comme une expérience quasiment virtuelle, et qui demande au spectateur de porter des lunettes spéciales pour bénéficier de l'effet 3D ? Ce que nous apprend cet exemple, c'est qu'aujourd'hui la figure de l'espace virtuel vient contaminer l'imaginaire tout entier. L'imaginaire est pensé d'emblée comme virtuel : immersion, déconnexion du réel environnant, expérience totale et englobante, persistante... Le virtuel deviendrait-il la norme pour l'expérience de l'imaginaire?

\section{Les espaces ubiquistes : un réel entre virtuel et actuel}

Mais la question est peut-être ailleurs. En effet, la réalité elle-même et non plus seulement l'imaginaire, est menacée de virtualisation. La réalité augmentée, I'ubimédia, sont aujourd'hui les nouvelles frontières du virtuel. Les futurologues nous prévoient pour demain un monde saturé d'information. La réalité virtuelle va envahir notre quotidien, en particulier pour jouer, et elle pourrait redéfinir « nos catégories mentales, notre identité, notre société, notre économie. Elle

6 L'épisode est relaté par exemple dans la version anglophone et numérique du grand quotidien israélien Ha'aretz: http://www.haaretz.com/news/in-pictures-palestinian-protesters-dress-as-avatar-s-na-vi-1.263316 (consulté le 2 janvier 2011) 
pourrait devenir le média de demain » (Sussan, 2009). Mais même dans le monde réel (au sens de : actuel), nous resterons branchés en permanence. L'informatique, après être devenue domestique via les PC, puis portable et nomade, via les laptops et les smartphones, va se répandre dans la plupart de nos objets quotidiens en poursuivant sa miniaturisation (Greenfield, 2007 ; Beau, 2008). Les nano-biotechnologies vont rendre les objets de notre environnement intelligents et communicants, et démultiplier nos capacités d'information et de communication, via de nouvelles interfaces toujours plus intégrées à nous-mêmes (De Rosnay 2008). A moyen ou long terme, nous attend la post-humanité, où l'homme fusionnerait avec la machine pour augmenter ses capacités, et peut-être devenir immortel (Sussan, 2005 ; Kurzweil, 2007).

A plus court terme, et de façon plus réaliste, la réalité augmentée permettrait par exemple " plaquer sur le réel un ensemble de données numériques" (Sussan, 2009). Ainsi, un individu évoluant dans une ville inconnue pourrait recevoir en temps réel, en semi-transparence sur des lunettes adaptées, des informations sur son environnement: voir ce qu'il y a à l'intérieur d'un bâtiment, afficher un itinéraire en surimpression sur le paysage urbain pour s'orienter vers une destination, obtenir des renseignements en temps réel sur un interlocuteur, savoir en permanence où se trouvent les membres de son réseau personnel, etc. Une partie de ces possibilités ne relèvent déjà plus de la science-fiction. Avec l'amélioration des systèmes de géolocalisation, des interfaces informatiques (écrans, commandes vocales, tactiles, ou par simple détection de mouvement), de la quantité de données disponibles sur les réseaux informatiques, de moteurs de recherche intelligents et personnalisés capable de trier, sélectionner et utiliser ces informations, le potentiel de la réalité augmentée est considérable. Les implications de cette révolution sont complexes et nombreuses, et viennent questionner les notions même de réalité, de perception, d'environnement, de rapports humains, de connaissance. La réalité sera virtuellement augmentée... ou ne sera pas. Et la distinction entre entre réel, actuel, virtuel et imaginaire, va devenir de plus en plus floue - et peut-être sans importance.

\section{Conclusion}

On le voit, l'espace virtuel entretient finalement des rapports complexes à la réalité, à l'actuel, à l'imaginaire. Les premiers espaces virtuels réels (hors de la fiction) ont été des simulateurs (de vol, de combat, de conduite, etc.), destinés à reproduire, à reconstituer la réalité, essentiellement dans un but d'entraînement et d'apprentissage. Une branche importante de la science-fiction, de P. K. Dick à Matrix, s'est passionnée pour cette fonction de l'espace virtuel comme simulacre de réalité : dans ces fictions, le monde entier se révèle parfois n'être qu'une illusion virtuelle. La notion de virtuel sert surtout à ces auteurs de prétexte à une réflexion métaphysique, voire mystique. Le titre original de Le Dieu venu du Centaure, de P. K. Dick, est The three stigmatas of Palmer Eldricth : ces "trois stigmates " renvoient bien sûr aux stigmates du Christ crucifié. Dans ce roman, le personnage de Palmer Eldritch parvient à capturer tous les autres personnages dans un univers mental artificiel qui n'est autre qu'une projection de son propre esprit, faisant de lui une sorte de Dieu omnipotent : on saisit par là toute l'ambition métaphysique de ce récit. On peut aussi, selon la formule d'Henri Desbois, voir dans un film comme Matrix comme « le versant populaire et vaguement new age de la critique "baudrillardienne" » (Desbois, 2006 : 133) : I'idée que le monde n'est qu'un simulacre, tant l'image, l'apparence, la communication finissent par supplanter la réalité.

L'espace virtuel intraficionnel, avec le cyberpunk, a été associé au début des années 80 à la 
révolution informatique, au point d'en épouser les formes, et d'apparaître comme un espace géométrique et abstrait, représentation pure des données numériques. Mais avec les progrès de la puissance de calcul, l'espace virtuel a fini par devenir un hyper-espace, un espace bigger than life où tout devient superlatif : les villes, les forêts, les paysages - et les aventures. Aujourd'hui, l'industrie numérique pioche dans la boîte à outil des archétypes et des représentations mythologiques pour créer des univers virtuels toujours plus réalistes et attractifs - jeux vidéos, films en 3D - au point d'arracher les jeunes générations aux autres supports de l'imaginaire, plus plats et moins virtuels, notamment le livre, mais peut-être bientôt l'image en 2D. Ces univers ont une telle densité qu'ils finissent par contaminer la réalité : celle-ci en vient à imiter la fiction virtuelle pour mieux s'adresser à notre imaginaire, comme le montrent les récupérations politiques d'Avatar. L'attraction de ces espaces virtuels peut s'avérer si forte que les Cassandre nous mettent en garde contre le risque d'addiction, de détachement de la réalité. Mais c'est pourtant à la réalité que nous ramène ce parcours dans les géographies virtuelles, avec l'émergence de la « réalité augmentée ", qui vient fusionner la réalité et son double numérique. Faut-il y voir la revanche du réel, ou au contraire la promesse de sa disparition, par dilution dans une virtualité triomphante ?

\section{Bibliographie}

Entre parenthèses, date de la première édition

Etudes:

BACHELARD G., 2009 (1957), La poétique de l'espace, Paris : PUF, col. Quadrige.

BAUDRILLARD J., 1981, Simulacres et simulations, Paris : Galilée.

BAUDRILLARD J., 1991, La guerre du golfe n'a pas eu lieu, Paris : Galilée.

BEAU F. (dir.), 2008, Cultures d'Univers : jeux en réseau, mondes virtuels, le nouvel âge de la société numérique, Limoges : FYP.

BERTHELOT F., CLERMONT P. (dir.), 2007, Colloque de Cerisy : science-fiction et imaginaires contemporains, Paris : Bragelonne, col. Essais.

BICAÏS M., 2007, "L'imaginaire colonisé par le dogme de la ressemblance ", in BERTHELOT F., CLERMONT P. (dir.), 2007, Colloque de Cerisy : science-fiction et imaginaires contemporains, Paris : Bragelonne, col. Essais, 39-52.

BOZETTO R., MENEGALDO G. (dir.), 2006, Colloque de Cerisy : les nouvelles formes de la sciencefiction, Paris : Bragelonne, col. Essais.

CALVET Y., 2006, "La persistance des mythes dans la science-fiction au cinéma ", dans BOZETTO Roger, MENEGALDO Gilles (dir.), 2006, Colloque de Cerisy : les nouvelles formes de la sciencefiction, Paris : Bragelonne, col. Essais, 237-254.

CASTELLS M., 1999, L'ère de l'information, Paris : Fayard, 3 vol. 
COLON É., LANGLET I., 2007, « Imaginaires urbains : de Los Angeles à Solotol », dans BERTHELOT Francis, CLERMONT Philippe (dir.), 2007, Colloque de Cerisy : science-fiction et imaginaires contemporains, Paris : Bragelonne, col. Essais, 135-151.

COLSON R., RUAUD A.-F., 2008, Science-fiction : les frontières de la modernité, Paris : Mnémos.

COULOMBE M., 2010, Le monde sans fin des jeux vidéo, Paris : PUF, col. La nature humaine.

DE CAYEUX A., GUIBERT C. (dir.), 2007, Second Life : un monde possible, Paris : Les petits matins.

DE ROSNAY J., 2008, 2020 : les scénarios du futurs, Paris : Fayard.

DESBOIS H., 2006, "Présence du futur : le cyberespace et les imaginaires urbains de sciencefiction ", Géographie et Cultures, $n^{\circ} 61,123-140$.

DESCARTES R., 1993 (1641), Méditations Métaphysiques, Paris : Garnier Flammarion, col. Philosophie.

DUPEYRON-LAFAY F., HUFTIER A. (dir.), 2007, Poétique(s) de l'espace dans les oeuvres fantastiques et de science-fiction, Paris : Michel Houdiard éditeur.

ECO U., 1985, La guerre du faux, Paris : Grasset.

ENGELIBERT J.-P., 2006, " Mondes clos et clôtures formelles. D'un rapport minoritaire au genre dans The Truman Show, Gattaca et Existenz ", dans BOZETTO R., MENEGALDO G. (dir.), 2006, Colloque de Cerisy : les nouvelles formes de la science-fiction, Paris : Bragelonne, col. Essais, 339350.

FORTIN T., MORA P., TREMEL L., 2006, Les jeux vidéos : pratiques, contenus et enjeux sociaux, Paris : L'Harmattan.

FORTIN T., TREMEL L., 2009, Mythologie des jeux vidéo, Paris : Le cavalier bleu.

GENETTE G., 1972, Figures III, Paris : Seuil, col. Poétique.

GENVO S. (dir.), 2006, Le game design de jeux vidéos : approches de l'expression vidéo-ludique, Paris : L'Harmattan.

GENVO S., 2009, Le jeu à son ère numérique : comprendre et analyser les jeux vidéo, Paris : L'Harmattan.

GREENFIELD A., 2007, Every[ware] : la révolution de l'ubimédia, Limoges, FYP.

JOURDE P., 1991, Géographies imaginaires : de quelques inventeurs de mondes au XXe siècle Gracq, Borges, Michaux, Tolkien, Paris : José Corti.

KURZWZEIL R., 2007, Humanité 2.0 : la bible du changement, Paris : M21 Editions. 
LABBÉ D., MILLET G., 2001, La science-fiction, Paris : Belin, col. Sujet.

LEVY P., 1998, Qu'est-ce que le virtuel ?, Paris : La découverte.

LANGLET I. 2006, La science-fiction : lecture et poétique d'un genre littéraire, Paris : Armand Colin, col. U Lettres.

MAC LUHAN M., 1977 (1964), Pour comprendre les médias, Paris : Seuil, col. Points.

MANFREDO S., 2000, La science fiction : aux frontières de I'homme, Paris : Gallimard, col. Découvertes.

MINNE S., 2007, "Le rêve entre fiction et métafiction : les mondes intrafictionnels ", dans BERTHELOT Francis, CLERMONT Philippe (dir.), 2007, Colloque de Cerisy : science-fiction et imaginaires contemporains, Paris : Bragelonne, col. Essais, 251-261.

MUSSET A., 2005, De New-York à Coruscant, Paris : PUF.

PLATON, 2002 (s.d.), La République, Paris : Garnier Flammarion, col. Philosophie.

SUSSAN R., 2005, Les utopies post-humaines : contre-culture, cybercultre, culture du chaos, Paris : Omniscience.

SUSSAN R., 2009, Demain, les mondes virtuels, Limoges, FYP.

VIRILIO P., 2010 (1996), Cybermonde, la politique du pire, Paris : Textuel.

\section{Romans:}

ASIMOV I., 2009 (1951), Fondation, Paris : Gallimard, col. Folio SF.

BANKS I., 2005 (1990), L'usage des armes, Paris : Robert Laffont.

BIOY CASARES A., 1992 (1940), L'invention de Morel, Paris : 10x18.

DICK P. K.., 2002 (1965), Le Dieu venu du centaure, Paris : J'ai Lu.

DICK P. K.., 1999 (1966), Ubik, Paris : 10x18.

GIBSON W., 2009 (1984), Neuromancien, Paris : J'ai Lu.

LIGNY J.-M., 1996, Inner City, Paris : J'ai Lu.

SIMMONS D.,1999 (1989), Hyperion, Paris : Robert Laffont, col. Ailleurs et Demain. 
STEPENSON N., 2000 (1992), Le samouraï virtuel, Paris : Le livre de poche.

THUILLIER G., 1999, Le dixième cercle, Paris : J'ai Lu.

TOLKIEN J. R. R., 2002 (1937), Bilbo le Hobbit, Paris : Christian Bourgois.

TOLKIEN J. R. R., 2003 (1955), Le seigneur des anneaux, Paris : Christian Bourgois.

\section{Films:}

AMENABAR A. 1997, Ouvre les yeux, Canal+ España / Las Producciones del Escorpión S.L. / Les Films Alain Sarde / Lucky Red / Sociedad General de Televisión (Sogetel).

BECKER W., 2003, Good Bye Lenin !, X-Filme Creative Pool.

BIGELOW K., 1995, Strange days, Lightstorm Entertainment.

CAMERON J.,2009, Avatar, $20^{\text {th }}$ Century Fox / Lightstorm Entertainment.

COPPOLA F. F., 1979, Apocalypse Now, Zoetrope Studios.

GILLIAM T., 1985, Brazil, Embassy International Pictures.

LANG F., 1927, Metropolis, Universum Film (UFA).

LISBERGER S., 1982, Tron, Walt Disney Productions / Lisberger/Kushner.

LUCAS G., 1977-2005, La guerre des étoiles, Lucasfilm / 20 $0^{\text {th }}$ Century Fox Pictures.

MALICK T., 1998, La ligne rouge, Fox 2000 Pictures / Geisler-Roberdeau / Phoenix Pictures.

PROYAS A., 1998, Dark City, Mystery Clock Cinema / New Line Cinema.

SCOTT R., 1982, Blade Runner, The Ladd Company / Shaw Brothers / Warner Bros Pictures, Michael Deeley Production / Ridley Scott Productions.

VERHOEVEN P., 1990, Total Recall. Carolco Pictures / TriStar Pictures.

WACHOWSKI A. \& L., 1999, Matrix, Groucho II Film Partnerschip / Silver Pictures / Village Roadschow Pictures / Warner Bros Pictures.

WEIR P. 1998, The Truman Show, Paramount Pictures / Scott Rudin Productions. 